\title{
Complex geometrical optics and nonlinear absorption/gain effects
}

\author{
Pawel Berczynski \\ Institute of Physics, West Pomeranian University of Technology, Szczecin 70-310, Poland
}

Received March 07, 2016; accepted June 15, 2016; published June 30, 2016

\begin{abstract}
The paper presents a systematic analysis of the influence of nonlinear absorption/gain phenomena on a Gaussian wave field evolution in the Kerr type medium with additional consideration of initial light beam convergence and divergence. To perform an efficient analysis of joint contribution of initial curvature of a wave front and nonlinear absorption/gain effects on self-focusing/self-defocusing phenomena I propose to apply the method of complex geometrical optics (CGO) which at once reduces Gaussian beam diffraction and selfaction effects (including nonlinear absorption/gain) to the domain of ordinary differential equations (ODEs), which are base (output) equations. The description in output ODEs results in CGO's great superiority over well known analytic methods of nonlinear optics such as: the variational method and the method of moments which every time require solving Nonlinear Schrodinger Equation (NLS) by applying an integral variational procedure or virial theory to obtain equations describing the evolution of amplitude, beam width and wave front curvature, which happen to be identical with those obtained by the CGO method. The CGO method dealing with output ODEs is a timeconsuming physical approach compared to numerical methods of nonlinear and wave optics. CGO with output evolutionary ODEs enables one to apply basic mathematical computer software such as: Matlab/Octave, Mathcad and Mathematica commonly available at every university, college or secondary school nowadays.
\end{abstract}

Real optical media exhibit absorption, which changes the properties of light propagating in nonlinear optical fibres and modifying essentially the conditions to achieve stable propagation of electromagnetic spatially limited modes [1]. However, despite the presence of dissipation effects which can be both linear and nonlinear it is still possible to obtain stable solutions in the form of dissipative solitons propagating in loss/gain magneto-optics systems, semiconductor optical amplifiers, reaction-diffusion media and Bose-Einstein condensates [2].

Thus, let me analyze first the influence of a nonlinear dissipative/gain profile when a Gaussian beam propagates in a classical optical medium with cubic nonlinearity. In a nonlinear medium of the Kerr type, the relative permittivity $\varepsilon$ can be presented in the form:

$$
\varepsilon=\varepsilon_{0} \pm \varepsilon_{N L}|u|^{2}
$$

where, in general, both $\varepsilon_{0}=\operatorname{Re} \varepsilon_{0}+i \operatorname{Im} \varepsilon_{0}={ }^{R} \varepsilon_{0}+i^{I} \varepsilon_{0}$ and $\quad \varepsilon_{N L}=\operatorname{Re} \varepsilon_{N L} \pm i \operatorname{Im} \varepsilon_{N L}={ }^{R} \varepsilon_{N L} \pm i^{I} \varepsilon_{N L} \quad$ can be complex-valued parameters, this way defining linear ${ }^{I} \varepsilon_{0}$ and nonlinear absorption ${ }^{I} \varepsilon_{N L}$ or gain $-\left(i^{I} \varepsilon_{N L}\right)$. Quantity ${ }^{R} \varepsilon_{N L}$ represents self-focusing and $-{ }^{R} \varepsilon_{N L}$ http://www.photonics.pl/PLP describes the self-defocusing effect. The role and practical application of the self-defocusing effect in contemporary optical applications are demonstrated in the paper [3]. In Eq. (1) the quantity $u$ represents a complex wave field, which in the case of the CGO method is a Gaussian beam in the form:

$$
u(\rho, z)=A \exp \left[i k_{0}\left(\sqrt{\varepsilon_{0}} z+B(z) \rho^{2} / 2\right)\right],
$$

where $B=B(z)$ is a complex parameter changing along propagation distance ( $z$ axis), which allows me to pack two observables in a single complex function in the form:

$$
B=\operatorname{Re} B+i \operatorname{Im} B=\kappa(z)+\frac{i}{k_{0} w^{2}(z)},
$$

where $\kappa=\kappa(z)$ is the wave front curvature, $w=w(z)$ is the beam width, $k_{0}=\frac{\omega}{c}=\frac{2 \pi}{\lambda}$ is the linear wave number for the light beam propagating in the $z$ direction in vacuum and $\rho=\sqrt{x^{2}+y^{2}}$ is the distance from the $z$ axis in cylindrical coordinates $(\rho, z)$ defining the geometry of a 3D optical nonlinear medium. It should be also emphasized that within geometrical optics description, the CGO method included, the wave field $u=u(\rho, z)$ in Eq. (2) should represent an electromagnetic wave being localized in the vicinity of propagation direction, which in our case is described by the $\mathrm{z}$-axis in cylindrical coordinates $(\rho, z)$. Moreover, the localization of the wave field $u=u(\rho, z)$ along the fibre axis means that the Gaussian field in Eq. (2) should be spatially narrow along the $z$-axis, which suggests the usage of a paraxial approximation natural for the methodology of geometrical optics. This way the application of a paraxial approximation within the CGO method, which embraces some wave phenomena (Gaussian beam diffraction and nonlinear self-action effects though CGO equations eikonal and transport equations - are purely of the geometrical optics character [4]), lets me define the amplitude of the Gaussian beam to be dependent only on the propagation coordinate $z$. This way one obtains in Eq. (2) that $A=A(z)$. Moreover, the CGO method deals not only with complex eikonal $\psi=\sqrt{\varepsilon_{0}} z+B(z) \rho^{2} / 2$ 
determined by the complex parameter $B$ in Eq. (3), which embraces the evolution of two observables: a Gaussian beam width and a wave front curvature packet into a single complex function $B=B(z)$, but also with a complex amplitude $A=|A(z)| e^{-i \phi(z)}$, which includes the evolution of power flow (in our case expressed in Gaussian units) along the z-axis: $P=w^{2}(z)|A(z)|^{2} \mathrm{c} / 8 \quad$ (parameter $\mathrm{c}$ denotes the light velocity) together with an additional phase (Gouy phase) $\varphi=\varphi(z)$, which shows the difference between the wave motion of a plane wave and a spatially limited beam, such as a Gaussian wave field. Taking into account the permittivity profile defined in Eq. (1), one can notice that the wave number in nonlinear dissipative/gain media is complex-valued:

$$
k=k_{0} \sqrt{\varepsilon}=k_{0} \sqrt{\left({ }^{R} \varepsilon_{0}+i^{I} \varepsilon_{0}\right) \pm\left({ }^{R} \varepsilon_{N L} \pm i^{I} \varepsilon_{N L}\right)|u|^{2}},
$$

where $|u|^{2} \sim \exp \left(-\rho^{2} / w^{2}\right)$. But, when we neglect the influence of linear damping $\left({ }^{I} \varepsilon_{0}=0\right)$ determining the area on an absorption curve where the linear optical medium is transparent (see [5-7]), one can notice that despite the fact that the wave number $k$ defined in Eq. (4) is, in general, a complex-valued quantity, the propagation constant $k_{z}=k(\rho=0, z)$ (e.g. the component of a wave vector, where the maximum intensity is propagated) is real. Nowadays, there are many papers concerning nonlinear optics discussing the joint influence of linear and nonlinear absorption processes (embracing twophoton absorption phenomenon) on the self-focusing of a Gaussian light beam. For instance, a number of numerical examples describing and discussing this problem are presented in the paper [6]. However, in the author's opinion it is very difficult to capture, in fact, the role of nonlinear absorption in a nonlinear self-focusing medium when linear dissipation dominates over nonlinear, which is demonstrated by the results in paper [6]. Thus, in the author's opinion the only reasonable way to answer how a nonlinear absorption phenomenon influences selffocusing/self-defocusing effects is to neglect at once the influence of a linear absorption effect, searching for the area on the absorption curve where the linear optical medium is transparent. This way, neglecting the influence of linear damping and substituting ${ }^{I} \varepsilon_{0}=0$ into Eq. (1) the author of the present paper describes analytically the evolution of a Gaussian light beam propagating along the $z$ axis in a nonlinear self-focusing/ self-defocusing medium in the presence of (nonlinear) two-photon absorption. This way, when ${ }^{I} \varepsilon_{0}=0$ permittivity profile takes the following form:

$$
\varepsilon={ }^{R} \varepsilon_{0}+\left( \pm{ }^{R} \varepsilon_{N L}+i^{I} \varepsilon_{N L}\right)|u|^{2}
$$

The meaning and practical role of a two-photon absorption effect within the effect of spatial soliton formation in a nonlinear medium of the Kerr type are demonstrated in the paper [5]. Following the procedure described in the book [4], to obtain CGO ODEs evolutionary equations, the author derived the first complex Riccati equation for linear and nonlinear dissipative/gain media for function $B$ in the form:

$$
\sqrt{{ }^{R} \varepsilon_{0}} \frac{d B}{d z}+B^{2}=-\left({ }^{R} \varepsilon_{N L} \mp i^{I} \varepsilon_{N L}\right) \frac{|A(0)|^{2} w^{2}(0)}{w^{4}} .
$$

Taking into account the case when the permittivity profile describing light propagation in a nonlinear absorptive/gain fiber is equal to $\varepsilon={ }^{R} \varepsilon_{0}+\left( \pm{ }^{R} \varepsilon_{N L}+i^{I} \varepsilon_{N L}\right)|u|^{2}$ and substituting the complex eikonal $\psi=\sqrt{{ }^{R} \varepsilon_{0}} z+B(z) \rho^{2} / 2$ into the transport (intensity) equation:

$$
\operatorname{div}\left(A^{2} \nabla \psi\right)=0
$$

the following equation was obtained:

$$
\frac{d A}{d z} \frac{\partial \psi}{\partial z}+\left[\frac{1}{\rho} \frac{\partial}{\partial \rho}\left(\rho \frac{\partial \psi}{\partial \rho}\right)+\frac{\partial^{2} \psi}{\partial z^{2}}+\frac{k_{0}{ }^{I} \varepsilon_{N L}|u|^{2}}{2}\right] A=0 .
$$

Using paraxial approximation to above equation in Eq. (8), the author expands the wave field $|u|^{2}$ in the Taylor series in $\rho$ in the vicinity of the fiber symmetry $z$ axis up-to a quadratic term, which means that the Gaussian wave field $|u|^{2}$ is spatially narrow, which in turn is an indispensable assumption to describe light propagation within the regularities of geometrical optics, including the CGO method presented in this paper. Thus one can notice that the quadratic term in the Taylor expansion essentially dominates over the higher-order ones, resulting in the described electromagnetic field $|u|^{2}$ localized along the propagation (fibre) axis (z). With the CGO condition for the wave field $|u|^{2}$ to be spatially narrow, let us substitute in Eq. (8) the radial coordinate as equal to zero $\rho=0$. As a result, the following connection was obtained:

$$
w^{2}(z)|A(z)|^{2}=w^{2}(0)|A(0)|^{2} .
$$

Regarding the above expression, let us formulate the following regularity: Nonlinear absorption/gain effects do not influence the component of the Poynting vector along which the maximum of electromagnetic wave field (maximum of the intensity) propagates. In our case, the application of axial geometry in $(\rho, z)$ coordinates to describe electromagnetic problem when the Gaussian field propagates along fibre axis means that the z-component of the Poynting vector (averaged in time for harmonic time 
dependence $\left.e^{i \omega t}\right):\left\langle\vec{S}_{z}\right\rangle$ is conserved in a nonlinear optical medium despite the presence of the nonlinear absorption effect (for instance two-photon or multi-photon absorption). Taking into account Eq. (9), the CGO equation for the Gaussian beam width evolution in a selffocusing medium with a contribution of nonlinear (twophoton) absorption/gain effects takes the form:

$$
{ }^{R} \varepsilon_{0} \frac{d^{2} w}{d z^{2}}=\frac{4\left(1-k_{0}^{2 R} \varepsilon_{N L}|A(0)|^{2} w^{2}(0)\right) \pm k_{0}^{4 I} \varepsilon_{N L}^{2}|A(0)|^{4} w^{4}(0)}{4 k_{0}^{2} w^{3}},
$$

where $w(0)=w(z=0)$ is the initial width and $A(0)=A(z=0)$ is the initial complex amplitude of a wave field. It is worth presenting Eq. (10) describing the evolution of dimensionless beam width $f=w / w(0)$ :

$$
\frac{d^{2} f}{d z^{2}}=-\frac{1}{f^{3}}\left(\frac{1}{L_{N L}^{2}}-\frac{1}{L_{D}^{2}} \mp \frac{{ }^{I} \varepsilon_{N L}^{2} k_{0}^{2}|A(0)|^{4}}{4^{R} \varepsilon_{0}}\right),
$$

defining: diffraction distance $L_{D}=k_{0} \sqrt{{ }^{R} \varepsilon_{0}} w^{2}(0)$ and nonlinear length in the form:

$$
L_{N L}=w(0) \sqrt{{ }^{R} \varepsilon_{0}} / \sqrt{{ }^{R} \varepsilon_{N L}|A(0)|^{2}} .
$$

To modify Eq. (11) to a more representative form, the author applies the relation:

$$
\frac{L_{D}^{2}}{L_{N L}^{2}}=\frac{P}{P_{c r i t}}
$$

where $P=\frac{1}{8} c \sqrt{{ }^{R} \varepsilon_{0}} w^{2}(0)|A(0)|^{2}$ is the total beam power along the $z$-axis and $P_{c r i t}=\frac{1}{8} \frac{c \sqrt{{ }^{R} \varepsilon_{0}}}{k_{0}^{2 R} \varepsilon_{N L}}$ is the critical power when the Gaussian beam propagates along the reference trajectory - fiber axis. Let us recall that when the total beam power is equal to critical, the Gaussian wave field collapses in a nonlinear Kerr type medium without any contribution of dissipation/gain effects. Using Eq. (12), the analytical solution of Eq. (11) has the following form:

$$
f^{2}=\frac{z^{2}}{L_{D}^{2}}\left(\Re^{2}(0) L_{D}^{2}-\frac{P}{P_{\text {crit }}}+1 \pm L_{D}^{2} \frac{{ }^{I} \varepsilon_{N L}^{2} k_{0}^{2}|A(0)|^{4}}{4}\right)+2 \Re(0) z+1
$$

where

$$
\mathfrak{R}(0)=\kappa(0) \pm k_{0}{ }^{I} \varepsilon_{N L}|A(0)|^{2} .
$$

The parameter $\mathfrak{R}(0)$ determines the joint influence of two processes: initial wave front curvature and nonlinear absorption/gain effects on Gaussian wave field evolution. These two effects can be at once included by introduction of a single convenient quantity $\mathfrak{R}(0)$, which simplifies essentially the wave description in nonlinear active/ dissipative optical media. The solution in Eq. (13) can be written next in a more convenient algebraic form:

$$
f^{2}=\left(\frac{\Re(0)}{\sqrt{\varepsilon_{0}}} z+1\right)^{2}+\left[1-\frac{P}{P_{\text {crit }}} \pm L_{D}^{2} \frac{{ }^{I} \varepsilon_{N L}^{2} k_{0}^{2}|A(0)|^{4}}{4}\right] \frac{z^{2}}{L_{D}} .
$$

One can notice that a stationary solution is possible when

$$
\kappa(0)=\mp k_{0}{ }^{I} \varepsilon_{N L}|A(0)|^{2} \text {. }
$$

This means that the wave mode possesses some specific properties when it propagates in nonlinear dissipative/gain media. Namely, the stationary wave mode propagates in a nonlinear absorptive/gain medium having a curved wave front which curvature is amplitude dependent. From Eq. (15) the author determines also the self-trapping power for the Gaussian wave mode as equal to:

$$
P=P_{c r i t}\left[1 \pm L_{D}^{2} \frac{{ }^{I} \varepsilon_{N L}^{2} k_{0}^{2}|A(0)|^{4}}{4}\right]
$$

The CGO solution for a self-defocusing medium has the form:

$$
f^{2}=\left(\frac{\Re(0)}{\sqrt{\varepsilon_{0}}} z+1\right)^{2}+\left[1+\frac{P}{P_{\text {crit }}} \pm L_{D}^{2} \frac{{ }^{I} \varepsilon_{N L}^{2} k_{0}^{2}|A(0)|^{4}}{4}\right] \frac{z^{2}}{L_{D}} .
$$

\section{References}

[1] N.N. Akhmediev, A. Ankiewicz, Solitons. Nonlinear Pulses and Beams (Chapman and Hall, London 1997).

[2] N.N. Akhmediev, A. Ankiewicz, Dissipative Solitons. Lecture Notes in Physics, 661, 1-443, ISBN: 978-3-540-23373-2 (Springer Berlin Heidelberg 2005).

[3] E. Jaatinen, M.W. Jones, Opt. Comm. 281, 3201 (2008).

[4] P. Berczynski, S. Marczynski, "Gaussian Beam Propagation in Inhomogeneous Nonlinear Media. Description in Ordinary Differential Equations by Complex Geometrical Optics" in P.W. Hawkes, Advances in Imaging and Electron Physics 185, 1111, ISBN: 9780128001448 (Elsevier New York 2014).

[5] Y. Silberberg, Opt. Letters 15(18), 1005 (1990).

[6] D. Ianetz, Yu. Kaganovskii, A.D. Wilson-Gordon, M. Rosenbluh, Phys. Rev. A 81, 53851 (2010).

[7] D. Mc Morrow, W. Lotshaw, J. Melinger, S. Buchner, R. Pease, IEEE Trans. Nucl. Sci. 49(6), 3002 (2002) 